\title{
Revisiting photocarrier lifetimes in photovoltaics
}

To the Editor - Recently, Pivrikas et al. published a Correspondence regarding the use of photocarrier lifetime in photovoltaic systems ${ }^{1}$. They proposed that the photocarrier lifetime in non-crystalline systems, which typically follows a Langevin-type recombination mechanism, should be treated not as a material parameter but rather a device parameter that depends on the experimental conditions. In contrast, the photocarrier lifetime can be used as a material parameter in crystalline (non-Langevin) systems. We agree with the authors about the importance of highlighting the correct use of photocarrier lifetimes. However, we disagree with the assertion that metal halide perovskites are non-crystalline and thus should be considered as Langevin systems. We argue that the effective photocarrier lifetime in all systems (Langevin or non-Langevin) in which electrons and holes recombine bimolecularly should be considered in the context of the experimental conditions, most importantly the photoexcited charge density.

Metal halide perovskites are generating increasing attention for their remarkable properties that enable their application in high-performance optoelectronics including solar cells, light-emitting diodes and photodetectors ${ }^{2}$. These materials are typically crystallized from halide salts as thin films or single crystals and adopt the $\mathrm{ABX}_{3}$ perovskite crystal structure, where $\mathrm{A}$ is an organic or caesium cation, $\mathrm{B}$ is a divalent metal cation and $\mathrm{X}$ is a halide anion. The resulting materials are predominantly crystalline as indicated by strong, oriented reflections from $\mathrm{X}$-ray diffraction measurements ${ }^{2}$.

The consensus from the scientific literature is that the dominant photoexcited species in these materials at solar illumination conditions are free electrons and holes as opposed to bound electron-hole pairs (excitons) ${ }^{3}$, which is consistent with recent reports of low exciton binding energies relative to thermal energy ${ }^{4}$. Importantly, bimolecular electron-hole recombination rates, $B$, are comparable to those in singlecrystalline inorganic semiconductors such as gallium arsenide, and these rates are many orders of magnitude lower than those predicted by the Langevin model ${ }^{5}$ $\left(B_{\mathrm{L}} / B>10^{4}\right)$, where $B_{\mathrm{L}}$ is the Langevin-type rate, even despite relatively modest carrier mobilities $^{6}\left(\approx 1-100 \mathrm{~cm}^{2} \mathrm{~V}^{-1} \mathrm{~s}^{-1}\right)$. These observations suggest that perovskites should be classified as non-Langevin systems, in contrast to that proposed by Pivrikas and colleagues. Nevertheless, we note that the correct description of charge carriers and of their transport and recombination mechanisms remain topics of intense study in the community ${ }^{7}$.

In general, the effective photocarrier lifetime in all systems (Langevin or nonLangevin) in which electrons and holes recombine bimolecularly will depend on the charge density. We agree with Pivrikas and colleagues that, in general, the bimolecular recombination rate is a more rigorous metric than photocarrier lifetime. However, while the bimolecular recombination constant is an intrinsic material property, it is the carrier lifetime that governs carrier dynamics, and in general the carrier lifetime arises not only from bimolecular recombination but will be influenced by other processes such as charge trapping. The measurement of carrier lifetime is thus a valuable experimental quantity that can be used to compare systems and extract other parameters for that given charge carrier density. For example, once the excitation density is defined, the diffusion length of charge carriers in materials such as perovskites extracted from photocarrier lifetime measurements is a valid quantity for further modelling and understanding ${ }^{5,8}$. We note that excitation intensities much higher than solar illumination conditions are often required for many spectroscopic techniques, imposing challenges on these methods to extract parameters relevant to the photovoltaic operating regime.

Optimizing photovoltaic materials such as perovskites by maximizing carrier lifetime is a useful procedure provided the lifetime is measured with photoexcitation intensities giving charge densities equivalent to those seen under solar illumination conditions. For example, a long carrier lifetime at open circuit is essential for building up an energized carrier population to maximize photovoltage. However, to reach the theoretical efficiency limits, it is also essential that this recombination is all radiative and any nonradiative decay is eliminated ${ }^{9}$. Measuring a long photocarrier lifetime alone, for example by time-resolved photoluminescence techniques, could be misleading as there may be very fast non-radiative decay pathways through which a large fraction of the carriers decay that are too fast to resolve. Furthermore, there is tendency in the perovskite community to place a large emphasis on long-lifetime tail components even though they might comprise only a tiny fraction of the total recombination pathways of the carriers. We propose that lifetime measurements should be conducted in conjunction with photoluminescence quantum yield measurements to ensure that the lifetime obtained is placed in the context of the material's emissivity and thus radiative limits for photovoltaics.

In conclusion, we argue that perovskites should be classified as crystalline and, given the current understanding, non-Langevin systems. We agree that the bimolecular recombination rate is a more universal parameter for all systems, though photocarrier lifetime for perovskites is still a useful metric for photovoltaic optimization and comparison to other systems provided photoexcited charge densities close to those achieved under solar illumination conditions are used. Although long lifetimes should be sought, perovskite photocarrier lifetime behaviour can be complicated and influenced by a number of factors including non-radiative recombination sites $^{5}$ and photon-recycling events ${ }^{10}$, so due care must be taken in these measurements to allow fair and rigorous assessment.

\footnotetext{
References

1. Pivrikas, A., Philippa, B., White, R. D. \& Juska, G. Nat. Photon. 10, 282-283 (2016).

2. Stranks, S. D. \& Snaith, H. J. Nat. Nanotech. 10, 391-402 (2015).

3. D'Innocenzo, V. et al. Nat. Commun. 5, 3586 (2014).

4. Miyata, A. et al. Nat. Phys. 11, 582-587 (2015).

5. Johnston, M. B. \& Herz, L. M. Acc. Chem. Res. 49, 146-154 (2016).

6. Brenner, T. M., Egger, D. A., Kronik, L., Hodes, G. \& Cahen, D. Nat. Rev. Mater. 1, 15007 (2016).

7. Zhu, X. Y. \& Podzorov, V. J. Phys. Chem. Lett. 6, 4758-4761 (2015).

8. Stranks, S. D. et al. Science 342, 341-344 (2013).

9. Miller, O. D., Yablonovitch, E. \& Kurtz, S. R. IEEE J. Photovolt 2, 303-311 (2012).

10. Pazos-Outón, L. M. et al. Science 351, 1430-1433 (2016).
}

Samuel D. Stranks ${ }^{1,2 \star}$ and Annamaria Petrozza ${ }^{3}$

${ }^{1}$ Cavendish Laboratory, JJ Thomson Avenue, Cambridge CB3 OHE, UK. ${ }^{2}$ Research Laboratory of Electronics, Massachusetts Institute of Technology, 77 Massachusetts Avenue, Cambridge, Massachusetts 02139, USA. ${ }^{3}$ Center for Nano Science and Technology@ PoliMi, Istituto Italiano di Tecnologia, Via Giovanni Pascoli 70/3, 20133 Milano, Italy. *e-mail: sds65@cam.ac.uk 